\title{
Electrocardiographic diagnosis of acute myocardial infarction in patients with implanted pacemakers
}

Sir:

Bathen and Abrahamsen (1973, 35, p. 1336) correctly note that, when acute myocardial infarction is suspected in a patient with an implanted pacemaker, the pacemaker should be deactivated to allow interpretation of the non-paced electrocardiogram. A practical consideration, which deserves emphasis, is that when pacing is terminated, prominent ST depressions and $T$ wave inversions simulating acute ischaemia or subendocardial infarction may be recorded. These changes occur most commonly in praecordial leads. Though their genesis is obscure, these abnormalities of repolarization secondary to pacing should be recalled particularly when they are occurring in the absence of chest pain or increases in enzyme levels.

Chatterjeee et al. (1969) first called attention to this phenomenon and Gould et al. (1973) later reported several additional cases. The duration of repolarization abnormalities is variable and appears to be correlated with the length of pacing and pulse amplitude. In one patient with a temporary pacemaker in whom there was intermittent pacing we noted that when the pacemaker was deactivated, subsequent sinus beats showed progressive diminution of $T$ wave inversions. This further attests to the fact that this phenomenon is indeed pacemakerrelated.

Michael A. Nevins, Bergen Pines County Hospital, Paramus, New Jersey 07652, U.S.A.

\section{References}

Chatterjee, K., Harris, A., Davies, G., and Leatham, A. (1969). Electrocardiographic changes subsequent to artificial ventricular depolarization. British Heart fournal, 31, 770.

Gould, L., Venkataraman, K., Goswami, M. K., and Gomprecht, R. F. (1973). Pacemaker-induced electrocardiographic changes simulating myocardial infarction. Chest, 63, 829. 